\title{
De novo chromosome 7q36.1q36.2 triplication in a child with developmental delay, growth failure, distinctive facial features, and multiple congenital anomalies: a case report
}

\author{
Muna A. Al Dhaibani', Diane Allingham-Hawkins ${ }^{2}$ and Ayman W. El-Hattab ${ }^{3^{*}}$ (D)
}

\begin{abstract}
Background: Studying human genome using chromosomal microarrays has significantly improved the accuracy and yield of diagnosing genomic disorders. Chromosome $7 \mathrm{q} 36$ deletions and duplications are rare genomic disorders that have been reported in a limited number of children with developmental delay, growth retardation, and congenital malformation. Altered dosage of $\mathrm{SHH}$ and $H L X B 9$, both located in 7q36.3, is believed to play roles in the phenotypes associated with these rearrangements. In this report we describe a child with $7 q 36.1 \mathrm{q} 36.2$ triplication that is proximal to the 7q36.3 region. In addition to the clinical description, we discuss the genes located in the triplicated region.

Case presentation: We report a 22 month old male child with a de novo $1.35 \mathrm{Mb}$ triplication at 7q36.1q36.2. His prenatal course was complicated by oligohydramnios, intrauterine growth restriction, and decreased fetal movement. Hypotonia, respiratory distress, and feeding difficulty were observed in the neonatal period. He also had developmental delay, cardiovascular malformation, growth failure with microcephaly, short stature, and underweight, sensorineural hearing loss, myopia, astigmatism, cryptorchidism, hypospadias, microphallus, lower extremity length discrepancy, bifid uvula, single palmer creases, and distinctive facial features including straight eyebrows, ptosis, up-slanted palpebral fissures, broad nasal bridge, low-set and posteriorly rotated ears, small mouth with thick lower lip, microretrognathia, and high-arched palate.

Conclusions: The child presented here had developmental delay, distinctive facial features, multiple congenital anomalies, and 7q36.1q36.2 triplication. This triplication, which was found to be de novo, has not been previously described and is believed to result in the observed phenotype. The triplicated region harbors the GALNTL5, GALNT11, KMT2C, XRCC2, and ACTR3B genes. GALNT11 encodes a membrane-bound polypeptide N-acetylgalactosaminyltransferase that can O-glycosylate NOTCH1 leading to the activation of the Notch signaling pathway. Therefore, increased GALNT11 dosage can potentially alter the Notch signaling pathway explaining the pathogenicity of 7q36 triplication. Studying further cases with similar genomic rearrangements is needed to make final conclusions about the pathogenicity of this triplication.
\end{abstract}

Keywords: Chromosome 7q36, Genomic rearrangements, Chromosomal microarray, Chromosomal disorders

\footnotetext{
* Correspondence: elhattabaw@yahoo.com

${ }^{3}$ Division of Clinical Genetics and Metabolic Disorders, Pediatric Department,

Tawam Hospital, Al-Ain, United Arab Emirates

Full list of author information is available at the end of the article
} 


\section{Background}

Studying human genome using chromosomal microarrays has significantly improved the accuracy and yield of diagnosing genomic disorders. Therefore, chromosomal microarrays have been routinely used as a first-tier diagnostic test for individuals with neurodevelopmental disabilities or congenital anomalies [1]. Compared to the $3 \%$ diagnostic yield of karyotyping, chromosomal microarray can detect pathogenic copy number variations (CNVs) with a diagnostic yield of 10-20\% [2, 3].

Chromosome 7 q36 rearrangements are rare genomic disorders. To date, deletions of $7 q 36$ have been described in 47 individuals with developmental delay and growth retardation. Other common features in these individuals are microcephaly, ear malformation, cleft lip and palate, holoprosencephaly, and sacral malformation. Haploinsufficiency of the genes $S H H$ and $H L X B 9$, both located in 7q36.3, are believed to be responsible for the holoprosencephaly and sacral malformation, respectively [4]. Duplications involving the $7 q 36.3$ region have been less frequently reported. Four individuals from a threegeneration family were reported with 7q36.3 duplication and intellectual disability, corpus callosum agenesis, Chiari malformation, macrocephaly, and distinctive facial features [5]. Additionally, 7q36.3 duplications were reported in two siblings with muscle hypertrophy and an infant with encephalocele [6, 7]. Although $S H H$ overexpression has been proposed to be responsible for the observed phenotypes in individuals with 7q36.3 duplications, the reason for heterogeneity in the phenotypes remains unclear [5].

In this report we describe a 7q36.1q36.2 triplication that is proximal to the $7 \mathrm{q} 36.3$ region and does not include $S H H$ in a child with developmental delay, growth failure, distinctive facial features, and multiple congenital anomalies.

\section{Case presentation}

A 22 months male child born to a second degree cousins who were healthy and had three healthy daughters. He was born at 35 week gestational age via a Cesarean section due to fetal distress. During pregnancy the mother had gestational diabetes that was diet controlled. The prenatal course was complicated by oligohydramnios, intrauterine growth restriction (IUGR), and decreased fetal movement noticed since the beginning of the third trimester. After birth, he was kept in neonatal intensive care unit (NICU) because of low birth weight $(1.6 \mathrm{~kg})$ and respiratory distress that required oxygen by nasal cannula for 2 weeks. He also had feeding difficulties required nasogastric tube feeding during the hospitalization. He was discharged home after 6 weeks when his oral feeding and respiratory distress improved.
He also had bilateral cryptorchidism, hypospadias, shallow scrotum, and microphallus. At the age of 3 months the stretched penile length was $1.7 \mathrm{~cm}$. At that age, sonography showed testes in inguinal canals, karyotype showed $46 \mathrm{XY}$, and hormonal studies showed normal testosterone, luteinizing hormone $(\mathrm{LH})$, thyroid function test, and insulin-like growth factor 1 (IGF-1). He received monthly testosterone injections for 3 months and the stretched penile length increased to $3.8 \mathrm{~cm}$ at the age of 6 months. At the age of 1 year he had bilateral orchidopexy.

Bilateral sensorineural hearing loss was diagnosed at the age of 13 months when he had an auditory brainstem response (ABR) testing. Subsequently, he started using hearing aids. In addition, he had snoring and otorhinolaryngology examination showed adenoid hypertrophy. Therefore bilateral myrigotomy and adenoidectomy were performed at the age of 18 months. In addition, he had bilateral congenital ptosis and eye exam at the age of 1 year revealed myopia and astigmatism.

During the NICU stay an echocardiogram showed patent ductus arteriosus with left to right shunt, branch pulmonary artery narrowing, and small aortic arch distal to the brachiocephalic artery and descending aorta. During the first year of life, he had several episodes of respiratory distress that required three hospitalizations and he was diagnosed to have reactive airway disease.

He started talking when he was 20 months and at the time of this report he could say about 10 words. $\mathrm{He}$ started rolling over at age of 7 months, sitting unsupported at 8 months, standing holding objects at 18 months, and at the time of this report he was able to walk holding furniture. He could also grasp and move objects from hand to hand, and waive bye-bye. He also had hypotonia since neonatal period. He started receiving speech therapy and physical therapy since the age of 1 year, with which he showed good response with improvements in hypotonia and his motor and language development. Brain MRI was done at the age of 20 months and was normal.

He also had growth failure with all growth parameters below 5 th percentiles. He had been eating regular table food and had normal swallowing assessment at the age of 1 year.

On physical examination his weight was $9.2 \mathrm{~kg}$, length $77 \mathrm{~cm}$, and head circumference $44.5 \mathrm{~cm}$ (all at about 3 SD below means). Distinctive facial features were observed including: straight eyebrows, bilateral ptosis with the left side being more pronounced than the right, up-slanted palpebral fissures, broad nasal bridge, low-set and posteriorly rotated ears, small mouth with thick lower lip, microretrognathia, and high-arched palate with bifid uvula (Fig. 1). Single palmer crease was observed bilaterally and right lower extremity was longer than the left by $1 \mathrm{~cm}$. 

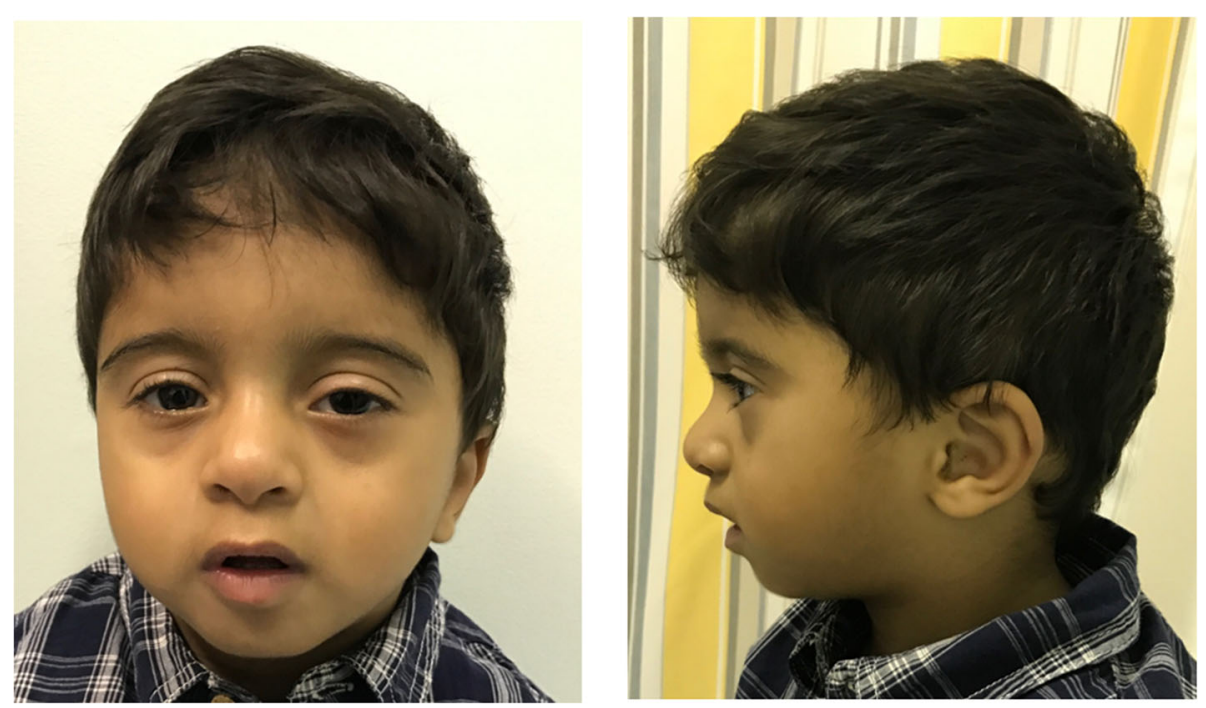

Fig. 1 Photograph showing the following distinctive facial features: straight eyebrows, bilateral ptosis with the left side being more pronounced than the right, up-slanted palpebral fissures, broad nasal bridge, low-set and posteriorly rotated ears, small mouth, thick lower lip, and microretrognathia

Chromosomal microarray did identify an $\sim 1.35 \mathrm{Mb}$ triplication at $7 \mathrm{q} 36.1 \mathrm{q} 36.2$, corresponding to a minimum triplication boundary of chr7:151,602,419-152,956,632 (hg19). Testing both parents confirmed that this triplication was de novo in child (Fig. 2). The CARE guidelines were followed in reporting this case.

\section{Discussion and conclusions}

Herein, we describe a 7q36.1q36.2 triplication in a boy with developmental delay, growth failure (microcephaly, short stature, and underweight), cardiovascular malformation (patent ductus arteriosus, branch pulmonary artery narrowing, and small aortic arch and descending aorta), sensorineural hearing loss, eye manifestations (ptosis, myopia, and astigmatism), genital anomalies (cryptorchidism, hypospadias, and microphallus), lower extremity length discrepancy, bifid uvula, single palmer creases, distinctive facial features (straight eyebrows, ptosis, up-slanted palpebral fissures, broad nasal bridge, low-set and posteriorly rotated ears, small mouth with thick lower lip, microretrognathia, and high-arched palate), prenatal complications (oligohydramnios, IUGR, and decreased fetal movement), and hypotonia, respiratory distress, and feeding difficulty observed in the neonatal period.

The 7q36 triplication in this child, which was found to be de novo, has not been previously reported. It is possible that increased dosage of one or more genes in the triplicated region is responsible for the observed phenotype. This region harbors 5 genes: GALNTL5, GALNT11, KMT2C, XRCC2, and ACTR3B. GALNTL5 and GALNT11 encode membrane-bound polypeptide
$\mathrm{N}$-acetylgalactosaminyltransferases. These enzymes catalyze O-glycosylation of peptides in the Golgi apparatus [8]. $K M T 2 C$ encodes a histone methyltransferase that can play a role in epigenetic transcriptional activation [9]. XRCC2 encodes a protein involved in homologous recombination repair of DNA damage. Biallelic mutations in this gene have been associated with Fanconi anemia, complementation group $\mathrm{U}[10]$. ACTR3B encodes an actin-related protein that may play a role in the organization of actin cytoskeleton. It is highly expressed in brain, heart, and white blood cells [11].

Among these five genes, increased dosage of KMT2C and $A C T R 3 B$ may be proposed to alter transcription and affect cytoskeleton, respectively, causing the pathogenicity of this triplication. However, we suggest that GALNT11 has the highest potential to be the candidate gene responsible for the observed phenotype because it was shown that the enzyme encoded by this gene can $\mathrm{O}$-glycosylates NOTCH1 leading to the activation of the Notch signaling pathway [12]. The Notch signaling pathway is a highly conserved cellular signaling system that plays a crucial role in metazoan development as it dictates cell fate through regulating differentiation, proliferation, and apoptosis, therefore influencing organ formation and morphogenesis. In mammals, the regulation of neurogenesis, myogenesis, angiogenesis, hematopoiesis, and epithelial-mesenchymal transition are all crucially influenced by Notch signaling. Defects in this pathway has been linked to a diverse group of human diseases including Alagille syndrome, Hajdu-Cheney syndrome, spondylocostal dysostoses type 1, CADASIL (cerebral arteriopathy with subcortical infarcts and 


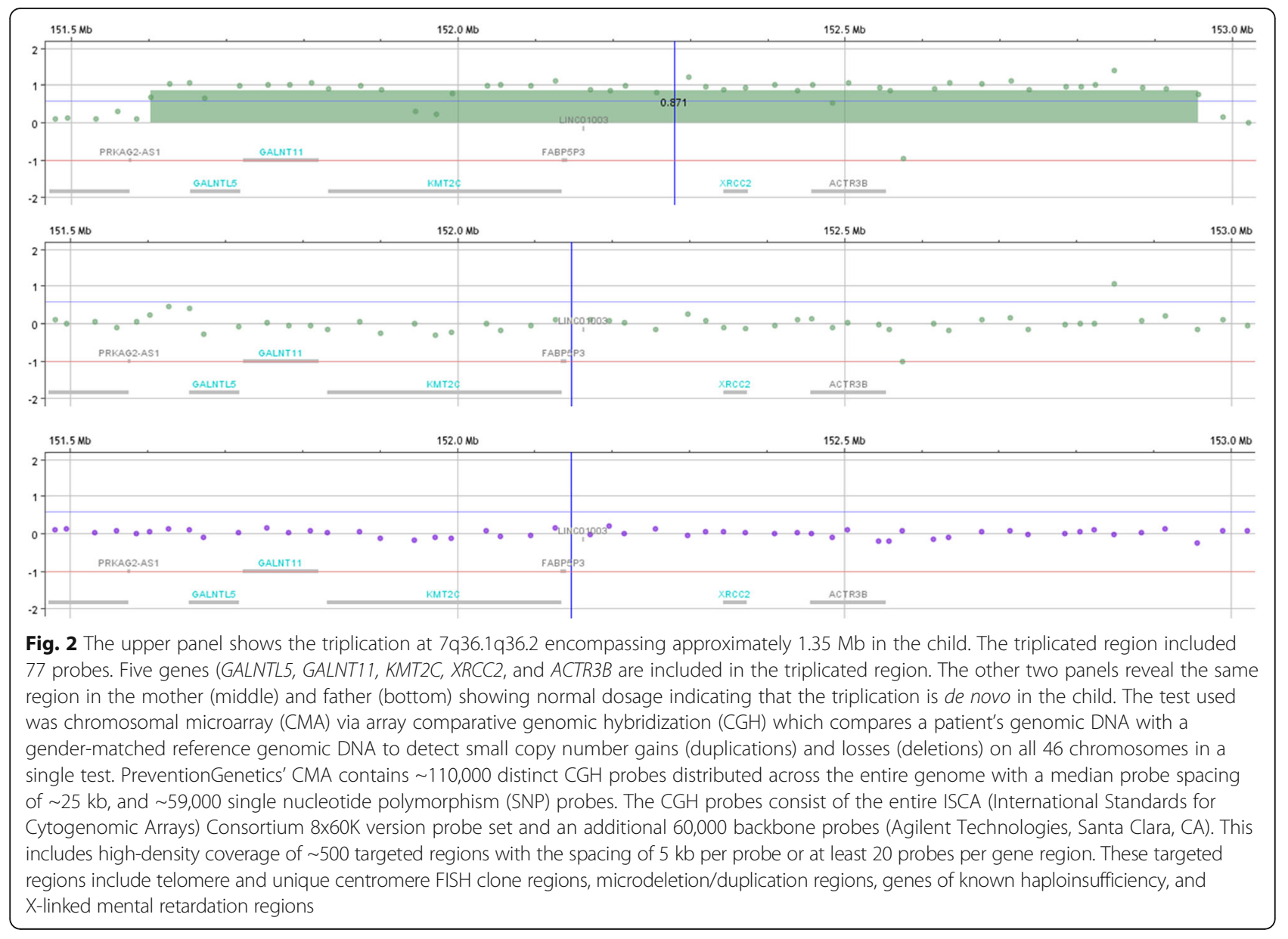

leukoencephalopathy), and Alzheimer disease type 3. Notch signaling is also widely implicated in somatic genomic mutational events leading to cancer and malignancy [13]. Therefore, increased GALNT11 dosage can potentially alter the Notch signaling pathway resulting in a widespread effects and multi-organ manifestations observed in this child with 7q36 triplication.

Although this triplication was found to be de novo and includes potential dosage-sensitive genes, it has not been previously described. Therefore, studying further cases with similar genomic rearrangements is needed before making final conclusions about the pathogenicity of this triplication. The likely mechanism for this triplication is non-allelic homologous recombination (NAHR) mediated by segmental duplications. Segmental duplications are estimated to comprise approximately $5 \%$ of the human genome and are predicted to facilitate the formation of deletions and duplications through unequal recombination between homologous regions [14]. The region of chromosome 7 involved in this case (7q36) has been reported to be flanked by segmental duplications that could mediate the formation of chromosomal rearrangements [15].
In summary, we present a boy with developmental delay, growth failure, cardiovascular malformation, sensorineural hearing loss, eye manifestations, genital anomalies, lower extremity length discrepancy, bifid uvula, and distinctive facial features who was found to have an $\sim 1.35 \mathrm{Mb}$ triplication at 7q36.1q36.2. The triplicated region includes GALNTL5, GALNT11, KMT2C, XRCC2, and ACTR3B. GALNT11 encodes a membrane-bound polypeptide $\mathrm{N}$-acetylgalactosaminyltransferase that can O-glycosylate NOTCH1 leading to the activation of the Notch signaling pathway. Therefore, increased GALNT11 dosage can potentially alter the Notch signaling pathway supporting the pathogenicity of $7 \mathrm{q} 36$ triplication.

\section{Acknowledgements}

We thank the child reported here and his family.

\section{Funding}

No funding was associated with this study.

Availability of data and materials

Microarray data is available at PreventionGenetics, LLC. Diane Allingham-Hawkins can be contacted at diane.allingham-hawkins@preventiongenetics.com in order to obtain the data. 


\section{Authors' contributions}

MAAD collected clinical data and participated in writing the manuscript. DA-H reviewed and summarized molecular data and participated in writing the manuscript. AWE-H evaluated the child, collected clinical and molecular data, and participated in writing the manuscript. All authors have read and approved the manuscript.

\section{Ethics approval and consent to participate}

The study was approved by Al-Ain Medical District Human Research Ethics Committee (15/89-CRD 416/15). Informed consent was obtained from parents.

\section{Consent for publication}

Informed consent was obtained from parents who consented to publish the images as well as the medical information supplied in this case report.

\section{Competing interests}

DA-H is an employee of PreventionGenetics, LLC.

\section{Publisher's Note}

Springer Nature remains neutral with regard to jurisdictional claims in published maps and institutional affiliations.

\section{Author details}

'Pediatrics Department, Tawam Hospital, Al-Ain, United Arab Emirates. ${ }^{2}$ PreventionGenetics, LLC, Marshfield, WI, USA. ${ }^{3}$ Division of Clinical Genetics and Metabolic Disorders, Pediatric Department, Tawam Hospital, Al-Ain, United Arab Emirates.

Received: 19 March 2017 Accepted: 17 October 2017

Published online: 23 October 2017

\section{References}

1. Miller DT, Adam MP, Aradhya S, Biesecker LG, Brothman AR, Carter NP, et al. Consensus statement: chromosomal microarray is a first-tier clinical diagnostic test for individuals with developmental disabilities or congenital anomalies. Am J Hum Genet. 2010;86(5):749-64.

2. Alabdullatif MA, Al Dhaibani MA, Khassawneh MY, El-Hattab AW. Chromosomal microarray in a highly consanguineous population: diagnostic yield, utility of regions of homozygosity, and novel mutations. Clin Genet. 2017;91(4):616-22.

3. Xiang B, Zhu H, Shen Y, Miller DT, Lu K, Hu X, et al. Genome-wide oligonucleotide array comparative genomic hybridization for etiological diagnosis of mental retardation: a multicenter experience of 1499 clinical cases. J Mol Diagn. 2010;12(2):204-12

4. Ayub S, Gadji M, Krabchi K, Côté S, Gekas J, Maranda B, et al. Three new cases of terminal deletion of the long arm of chromosome 7 and literature review to correlate genotype and phenotype manifestations. Am J Med Genet A. 2016;170A(4):896-907.

5. Wong K, Moldrich R, Hunter M, Edwards M, Finlay D, O'Donnell S, et al. A familial 7q36.3 duplication associated with agenesis of the corpus callosum. Am J Med Genet A. 2015;167A(9):2201-8.

6. Bear KA, Solomon BD, Roessler E, Alvarez DEP, Kubendran S, O'Hara M, et al. Evidence for $\mathrm{SHH}$ as a candidate gene for encephalocele. Clin Dysmorphol. 2012;21(3):148-51.

7. Kroeldrup L, Kjaergaard S, Kirchhoff M, Kock K, Brasch-Andersen C, Kibaek M, et al. Duplication of 7q36.3 encompassing the sonic hedgehog $(\mathrm{SHH})$ gene is associated with congenital muscular hypertrophy. Eur J Med Genet. 2012:55(10):557-60.

8. Peng C, Togayachi A, Kwon Y-D, Xie C, Wu G, Zou X, et al. Identification of a novel human UDP-GalNAc transferase with unique catalytic activity and expression profile. Biochem Biophys Res Commun. 2010;402(4):680-6.

9. Daniel JA, Santos MA, Wang Z, Zang C, Schwab KR, Jankovic M, et al. PTIP promotes chromatin changes critical for immunoglobulin class switch recombination. Science. 2010;329(5994):917-23.

10. Park J-Y, Virts EL, Jankowska A, Wiek C, Othman M, Chakraborty SC, et al. Complementation of hypersensitivity to DNA interstrand crosslinking agents demonstrates that XRCC2 is a Fanconi anaemia gene. J Med Genet. 2016;53(10):672-80.
11. Jay $P$, Bergé-Lefranc $J L$, Massacrier A, Roessler E, Wallis D, Muenke $M$, et al. ARP3beta, the gene encoding a new human actin-related protein, is alternatively spliced and predominantly expressed in brain neuronal cells. Eur J Biochem. 2000;267(10):2921-8.

12. Boskovski MT, Yuan S, Pedersen NB, Goth CK, Makova S, Clausen H, et al. The heterotaxy gene GALNT11 glycosylates notch to orchestrate cilia type and laterality. Nature. 2013;504(7480):456-9.

13. Hori K, Sen A, Artavanis-Tsakonas S. Notch signaling at a glance. J Cell Sci. 2013:126(Pt 10):2135-40.

14. Bailey JA, Gu Z, Clark RA, Reinert K, Samonte RV, Schwartz S, et al. Recent segmental duplications in the human genome. Science. 2002;297(5583):1003-7.

15. Rudd MK, Keene J, Bunke B, Kaminsky EB, Adam MP, Mulle JG, et al. Segmental duplications mediate novel, clinically relevant chromosome rearrangements. Hum Mol Genet. 2009;18(16):2957-62.

\section{Submit your next manuscript to BioMed Central and we will help you at every step:}

- We accept pre-submission inquiries

- Our selector tool helps you to find the most relevant journal

- We provide round the clock customer support

- Convenient online submission

- Thorough peer review

- Inclusion in PubMed and all major indexing services

- Maximum visibility for your research

Submit your manuscript at www.biomedcentral.com/submit 